\title{
Engagement-Scenario-Based Decoy-Effect Simulation Against an Anti-ship Missile Considering Radar Cross Section and Evasive Maneuvers of Naval Ships
}

\author{
Kookhyun Kim ${ }^{1}$ \\ ${ }^{I}$ Professor, School of Naval Architecture \& Ocean Engineering, Tongmyong University, Busan, Korea
}

KEY WORDS: Decoy effect, Radar cross section, Evasive maneuver, State-space model, Sea-skimming active radar homing missile

\begin{abstract}
The survivability of a naval ship is the ability of the ship and its onboard systems to remain functional and continue a designated mission in man-made hostile environments. A passive decoy system is primarily used as a weapon system for improving the survivability of a naval ship. In this study, an engagement scenario-based simulation program was developed for decoy effectiveness assessments against an anti-ship missile (ASM), which tracks a target with sea-skimming and active radar homing. The program can explain the characteristics of a target ship, such as the radar cross section and evasive maneuvers, as well as the operational performance of the onboard decoy system, the guidance method of the ASM, and the engagement environment's wind speed and direction. This paper describes the theory and formulations, configuration, and user interface of the developed program. Numerical examples of a decoy effect assessment of a virtual naval ship against an ASM are presented.
\end{abstract}

\section{Introduction}

The survivability of a naval ship is the ability of the ship and its onboard systems to remain functional and continue a designated mission in man-made hostile environments. The survivability can be categorized using three sub-parameters: susceptibility, vulnerability, and recoverability. The total survivability can be assessed combining the probability measures of the sub-parameters (Kim and Lee, 2014). Susceptibility is the probability of not avoiding being hit by threats like anti-ship missiles (ASMs) and depends on the performance of threats and anti-air warfare (AAW) systems against them, as well as their signature characteristics.

The signatures of naval ships, such as the radar cross section (RCS), infrared (IR) signature, and visual signature, directly influence the susceptibility to threats. Particularly, RCS of a target ship is a factor for determining the performance of passive or active decoy systems for onboard soft-kill warfare against ASMs that use active radar homing for targeting and tracking. The decoy effectiveness is used as an index for the performance of passive decoy systems. A cumulative Gaussian distribution model has been used as a simple tool for decoy effectiveness assessment (An and Seo, 2015) but cannot explain the variation in RCS and performance of a decoy system for various engagement scenarios.

There have been studies on engagement scenario-based simulations (TTI, 2002; Kumar, 1990; Manji et al., 2002). Chapman and Benke (2000) described a ship-to-air defense model (SADM) for susceptibility assessment in engagement environments, which includes soft-kill ability modeling with active decoys, chaff, and jammers; hard-kill ability modeling with missiles, guns, and firecontrol systems; and a command-and-control system to track targets and coordinate soft-kill and hard-kill responses. Dan et al. (2016) presented a modeling and simulation (M\&S) technique for a decoy system and its countermeasure capability against ASMs. Lukenbill (1990) and Swee (2000) simulated a target aircraft in an anti-air missile engagement scenario based on a state-space model and various guidance methods. Poulos (1994) studied anti-air warfare for a small navy group in defensive scenarios. Regarding the decoy effect, Seo et al. (2012) estimated the dynamic behavior and the corresponding RCS of decoy clouds in an engagement environment with wind conditions. Kim (2020) introduced a framework on a decoy effect simulation against an ASM to consider the RCS and tactical diameter of warships. In this research, an engagement scenario-based simulation program

Received 17 May 2021, revised 8 June 2021, accepted 14 June 2021

Corresponding author Kookhyun Kim: +82-51-629-1658, kimk@tu.ac.kr

(C) 2021, The Korean Society of Ocean Engineers

This is an open access article distributed under the terms of the creative commons attribution non-commercial license (http://creativecommons.org/licenses/by-nc/4.0) which permits unrestricted non-commercial use, distribution, and reproduction in any medium, provided the original work is properly cited 
named ASMD/RCS (ASM Defense/RCS) was developed for decoy effectiveness assessments. This program was implemented in MATLAB/Simulink with the assumption that ASMs are the seaskimming type and use an active radar homing for targeting and tracking (Kim, 2020). The theory and formulations were obtained from Swee (2000). ASMD/RCS can explain the RCS and evasive maneuvers of a target ship, the operational performance of an onboard passive-type decoy system, the tracking scheme of ASMs, and engagement environments, including the wind speed and direction. This paper describes details of the developed program, the theory and formulations, configuration, the scenario designation, and the user interface. A decoy effectiveness assessment of a virtual naval ship is presented as a numerical example.

\section{Theory and Formulations}

Countermeasures with a decoy system are a general means of avoiding being hit by ASMs. Traditionally, a passive-type decoy system has been used, which is a soft-kill method of guiding ASMs to a fake target that is generated with a decoy cloud instead of the real target ship (Fig. 1). The decoy effectiveness depends on the RCS characteristics of the target ship and the decoy system. Generally, the higher the difference in RCS level is between the target ship and the decoy, the lower the hit probability is. However, the characteristics of the ship, decoy, and engagement environments collectively influence the probability. Examples are the evasive maneuver and RCS pattern of the target ship, the deploying scheme (range and direction) and RCS of the decoy, the dynamic performance of the ASM, and environmental conditions, such as the wind speed, direction, etc. In this study, these characteristic parameters were considered for the decoy effect simulation in a more realistic engagement scenario.

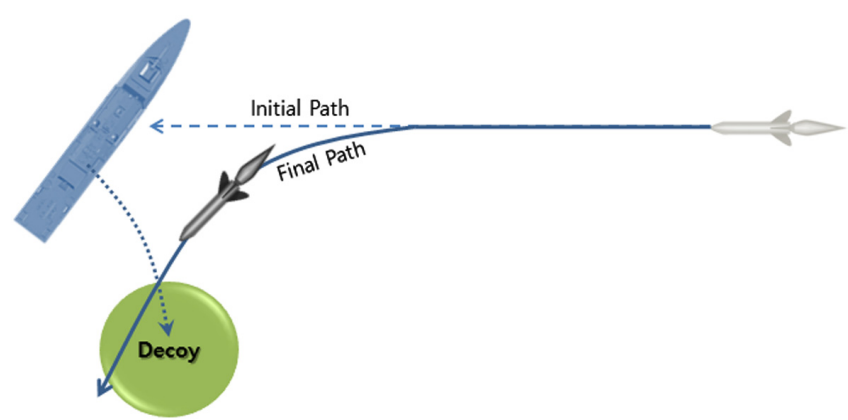

Fig. 1 Concept of soft-kill against ASMs using passive-type decoy system

\subsection{Target Ship Dynamics}

The decoy effectiveness against ASMs depends on the evasive maneuvers of the target ship, which are based on the turning radius of the ship, as shown in Fig. 2. In general, the turning radius of the ship depends on the rudder angle and the instantaneous moving speed. In this study, however, the turning radius was calculated in meters with Eq. (1).

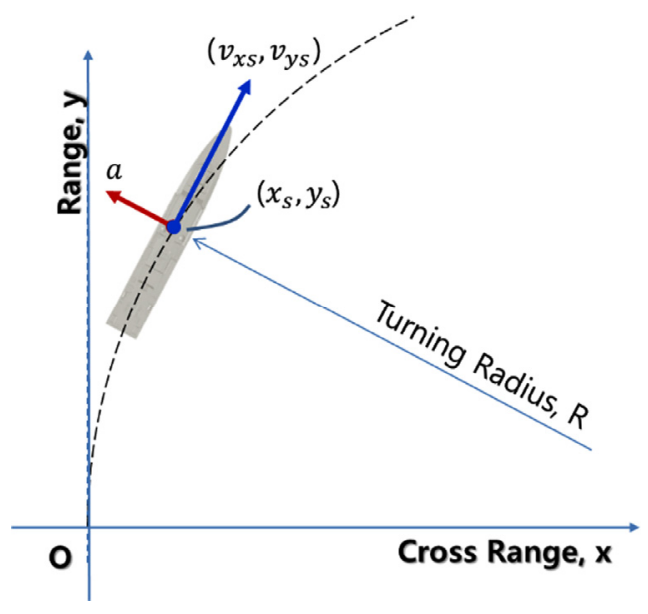

Fig. 2 Turning radius of the target ship for evasive maneuver against ASMs

$$
R=\frac{v_{s}^{2}}{a}=\frac{v_{s}^{2}}{g \cdot G_{s}^{*}}
$$

where $v_{s}=\sqrt{v_{x s}^{2}+v_{y s}^{2}}$ is the instantaneous tangential speed of the target ship in $\mathrm{m} / \mathrm{s}, \quad a=g \cdot G_{s}^{*}$ is the instantaneous centripetal acceleration in $\mathrm{m} / \mathrm{s}^{2}, g$ is gravitational acceleration $\left(9.81 \mathrm{~m} / \mathrm{s}^{2}\right)$, and $G_{s}^{*}=a / g$ is the g-value.

The target ship dynamics is implemented with the state-space equation below (Swee, 2000) based on the coordinate system in Fig. 2:

$$
\left\{\begin{array}{l}
\dot{x}_{s} \\
\dot{v}_{x s} \\
\dot{y}_{s} \\
\dot{v}_{y s}
\end{array}\right\}=\left[\begin{array}{cccc}
0 & 1 & 0 & 0 \\
0 & 0 & 0 & -\omega_{s} \\
0 & 0 & 0 & 1 \\
0 & \omega_{s} & 0 & 0
\end{array}\right]\left\{\begin{array}{c}
x_{s} \\
v_{x s} \\
y_{s} \\
v_{y s}
\end{array}\right\}
$$

where $x_{s}$ and $y_{s}$ are the coordinates of the cross range and the range in $\mathrm{m}$, respectively, $v_{x s}$ and $v_{y s}$ are the instantaneous speed of the target ship in $\mathrm{m} / \mathrm{s}, \omega_{s}=g \cdot G_{s}^{*} / v_{s}$ is the commanded evasive turning rate in $1 / \mathrm{s}$ of the target ship and is constant during the simulation, and means the time derivative of these variables. For reference, a constant g-value $G_{s}^{*}$ was used for the evasive maneuverability of the target ship instead of the centripetal acceleration. Negative and positive values mean starboard-side (right turn) and port-side evasive maneuvers (left turn) of the target ship, respectively.

\subsection{Target Ship RCS}

The inherent RCS pattern of the target ship influences the decoy effect on ASMs. The RCS pattern of the target ship varies with the azimuth angle, as shown in Fig. 3, which means that the RCS level also changes with the approach angle of the ASM, and the decoy effect varies with it as well. The predefined RCS pattern of the target ship was used to calculate the decoy RCS value of the azimuth angle corresponding to the ASM approach. 


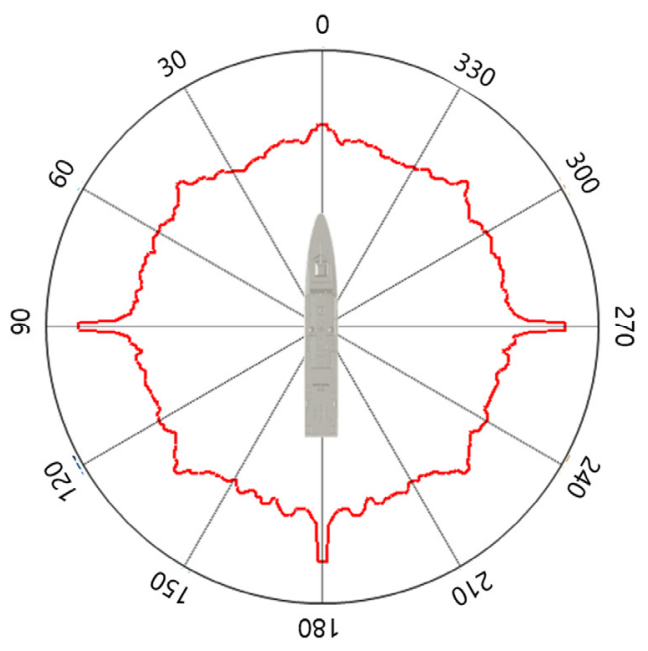

Fig. 3 RCS pattern of the target ship with azimuth angle

\subsection{ASM Dynamics}

The ability of an ASM to intercept a target ship mainly depends on the guidance method. Two main types of guidance methods can be employed: proportional navigation (PN) and command to line of sight (CLOS). PN is the most widely used homing guidance law and seeks to null the line-of-sight (LOS) angle rate by making the missile turn rate directly proportional to the LOS rate. CLOS uses only the angular coordinates between the ASM and the target ship, and the ASM is made to be in the LOS between the launcher and target. Any deviation of the missile from this line is corrected.

CLOS has inherent range limitations due to its sensitivity to angular tracking errors between the launching station and the ship target. PN is self-homing and relies on an on-board seeker to provide the target's LOS information directly, so it does not suffer from the range limitations encountered by CLOS (Swee, 2000). 2-dimensional missile dynamics was simulated by assuming an engagement scenario where the target ship does evasive maneuvers against sea-skimming ASMs that employ a PN homing law when tracking the ship target (Fig. 4).

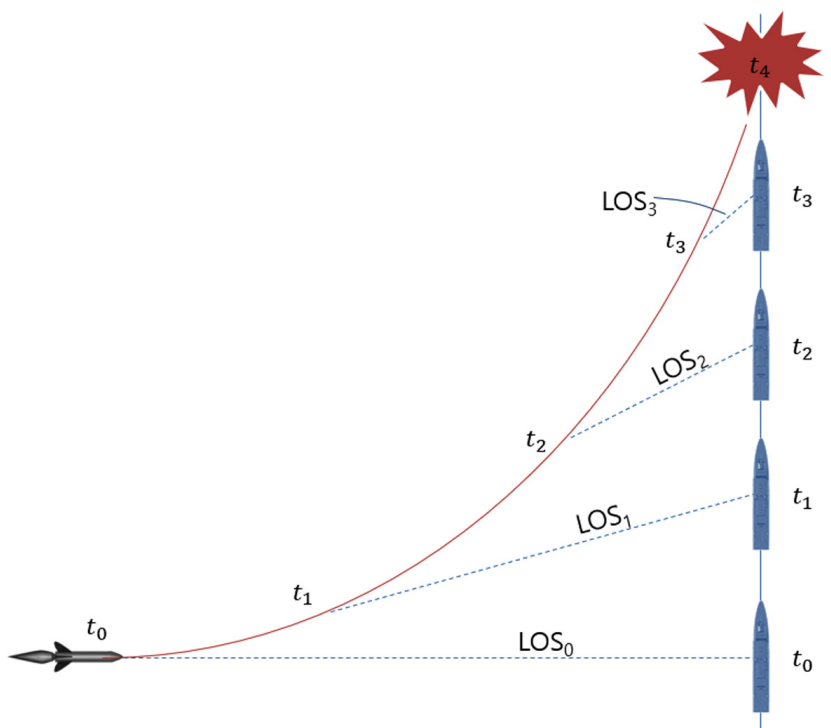

Fig. 4 Proportional navigation to intercept the ship target

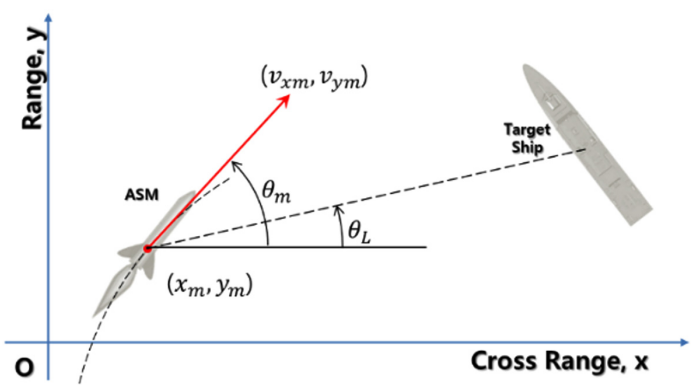

Fig. 5 ASM guidance to the target ship

Similarly to the target ship, the ASM dynamics can be implemented with the state-space equation below with the coordinate system in Fig. 5:

$$
\left\{\begin{array}{l}
\dot{x}_{m} \\
\dot{v}_{x m} \\
\dot{y}_{m} \\
\dot{v}_{y m}
\end{array}\right\}=\left[\begin{array}{cccc}
0 & 1 & 0 & 0 \\
0 & 0 & 0 & -\omega_{m} \\
0 & 0 & 0 & 1 \\
0 & \omega_{m} & 0 & 0
\end{array}\right]\left\{\begin{array}{c}
x_{m} \\
v_{x m} \\
y_{m} \\
v_{y m}
\end{array}\right\}
$$

where $x_{m}$ and $y_{m}$ are the coordinates of the cross range and the range in $\mathrm{m}, v_{x m}$ and $v_{y m}$ are the instantaneous speeds of the ASM in $\mathrm{m} / \mathrm{s}$, respectively, and $\omega_{m}$ is the commanded turning rate of the ASM in 1/s, which varies depending on the applied guidance method and state. Two types of PN can be applied: true- and pure-proportional navigation (TPN \& PPN). This study employs TPN, and the commanded turn-rate of the ASM is calculated by Eq. (4):

$$
\omega_{m}=\frac{N \cdot v_{c} \cdot \dot{\theta}_{L}}{\cos \left(\theta_{m}-\theta_{L}\right) \sqrt{v_{x m}^{2}+v_{y m}^{2}}}
$$

where $N$ is a proportional constant that typically varies from 3 to 5 (3 was used in this study). $v_{c}$ is the instantaneous closing velocity of the ASM to the target ship in $\mathrm{m} / \mathrm{s} . \dot{\theta}_{L}$ is the LOS rate between the ASM and the target ship in rad/s. $\theta_{m}$ and $\theta_{L}$ are the moving directions of the ASM and the LOS angle with respect to the cross-range axis in rad, respectively.

\subsection{Decoy Dynamics}

The decoy dynamics is a complex problem that is influenced by the engagement environment, including the wind speed and direction.

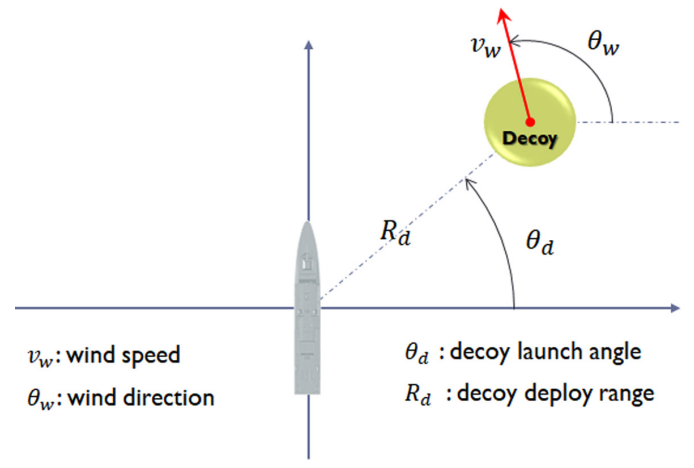

Fig. 6 Geometry of decoy dynamics 
Therefore, the problem should be simplified for convenience in the simulation. This study assumes that the decoy operates as follows: (1) launching to the direction $\theta_{d}$ in rad and deploying at the range $R_{d}$ in $\mathrm{m}$, (2) forming a decoy cloud and retaining its size, and (3) moving at the wind speed $v_{w}$ in $\mathrm{m} / \mathrm{s}$ along the wind direction $\theta_{w}$ in rad, where the decoy has an omni-directional scatter, and the RCS level is kept constant during the simulation (Fig. 6).

\subsection{Equivalent Scattering Center Targeting}

The RCS of the target ship can be represented with a scattering source point called a "scattering center" in the far field, which is when the target ship is electromagnetically far enough from the ASMs. Interestingly, in an engagement environment, an ASM intercepts the virtual scattering center, not the real target ship (Fig. 7(a)). Therefore, when the target ship deploys a decoy with the intended RCS level to the offboard range and direction, the scattering center moves along the target-to-decoy line (Fig. 7(b) and Fig. 7(c)). As a result, the LOS moves, and the susceptibility changes. The larger the decoy RCS is, the further the scattering center is from the target ship, and the lower the susceptibility is to the ASM.

(a)

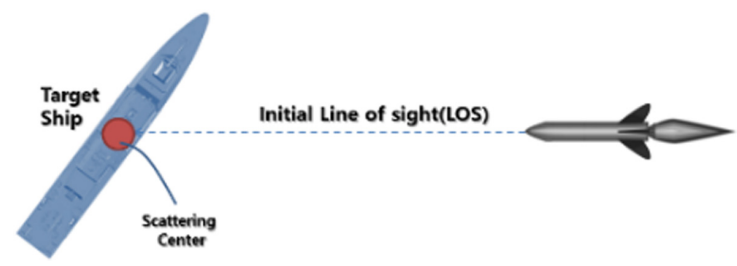

(b)

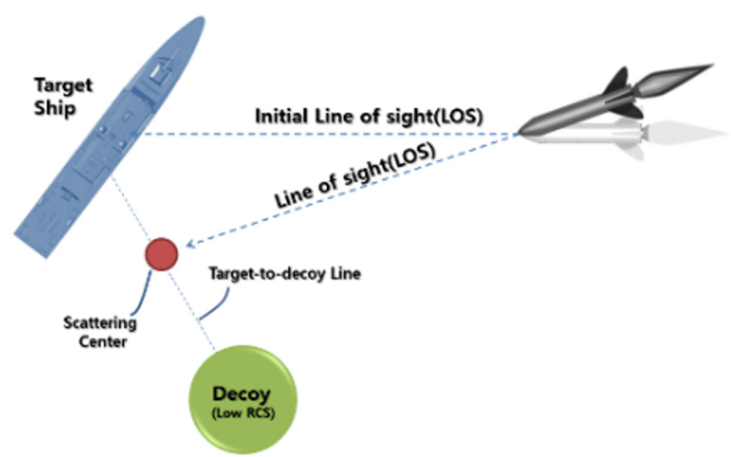

(c)

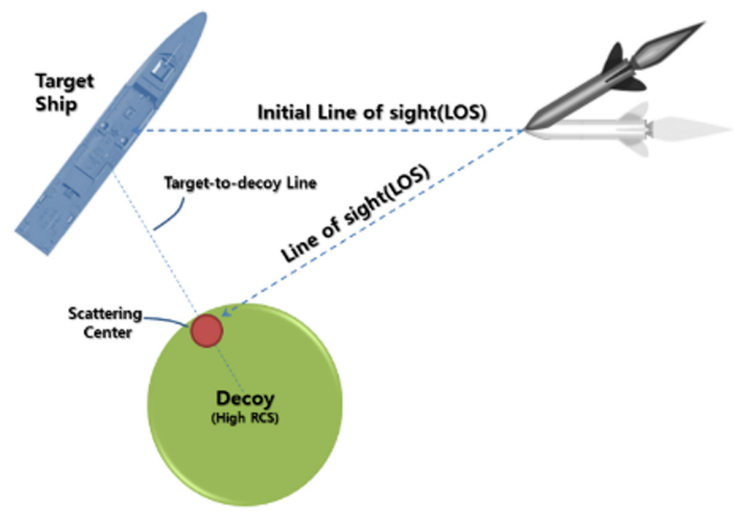

Fig. 7 Scattering center position change by decoy RCS level: (a) no decoy, (b) small decoy (w/ low RCS), and (c) large decoy (w/ high RCS)

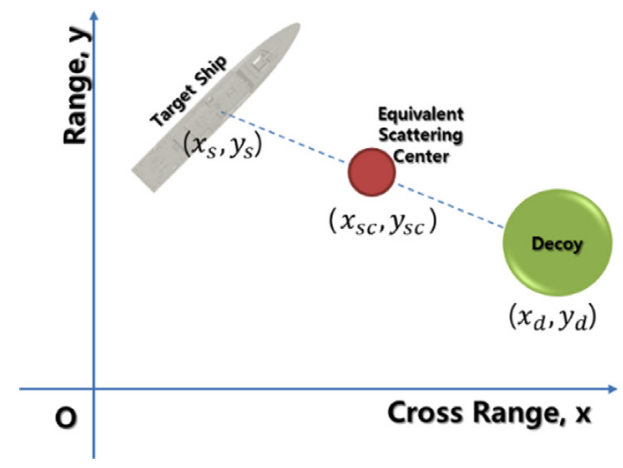

Fig. 8 Equivalent scattering center position

Referring to the coordinate system in Fig. 8, this study estimates the RCS level $\sigma_{s c}$ in dBsm and position $\left(x_{s c}, y_{s c}\right)$ in $\mathrm{m}$ of the equivalent scattering center, which is distinguished from that of the target ship using Eq. (5) and Eq. (6), respectively:

$$
\begin{gathered}
\sigma_{s c}=10 \log _{10}\left(10^{\sigma_{s} / 10}+10^{\sigma_{d} / 10}\right) \\
\left\{\begin{array}{l}
x_{s c}=\frac{10^{\sigma_{s} / 10} x_{s}+10^{\sigma_{d} / 10} x_{d}}{10^{\sigma_{s d} / 10}} \\
y_{s c}=\frac{10^{\sigma_{s} / 10} y_{s}+10^{\sigma_{d} / 10} y_{d}}{10^{\sigma_{s s} / 10}}
\end{array}\right.
\end{gathered}
$$

where $\sigma_{s}$ and $\sigma_{d}$ are the RCS level in dBsm of the target ship and the decoy cloud, respectively. $\left(x_{d}, y_{d}\right)$ is the instantaneous position of the decoy cloud in $\mathrm{m}$ and is determined by assuming that the deployed cloud moves along the wind direction with the same speed as the wind.

\section{ASMD/RCS}

ASMD/RCS was implemented using the theory and formulations presented in the previous section. ASMD/RCS can consider the performance of the target ship and the ASM, the engagement environments, and the way to simulate the decoy effectiveness (Fig.9).

The program consists of four blocks, as shown in Fig. 10: a decoy block, guide block, target ship block, and missile block. The decoy block includes the decoy performance and dynamics (RCS, deployment range and direction, and movement wind and its direction). The guide block implements the ASCM's targeting and tracking with the missile-to-target guidance algorithm. The target ship block includes the target ship performance and status (the initial

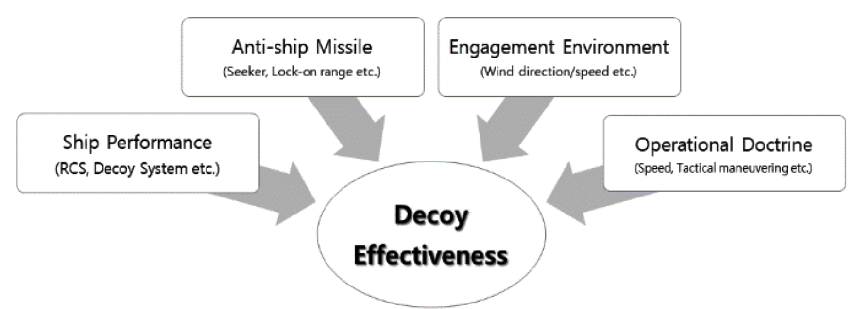

Fig. 9 Considerations of ASMD/RCS for decoy effectiveness assessment 
(a)

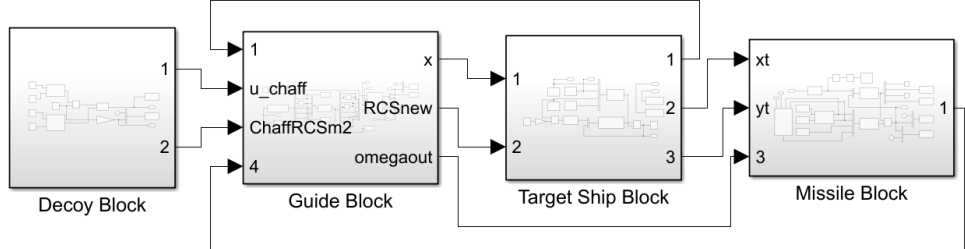

(b)

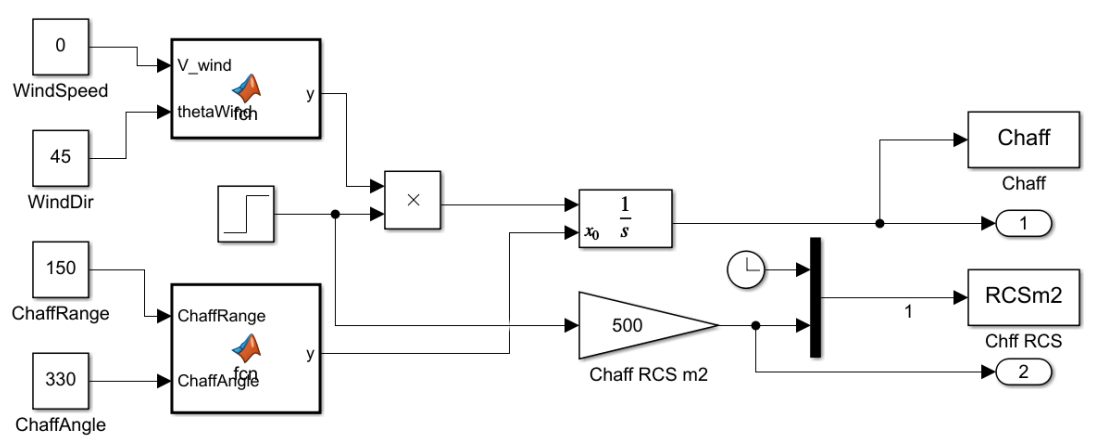

(c)

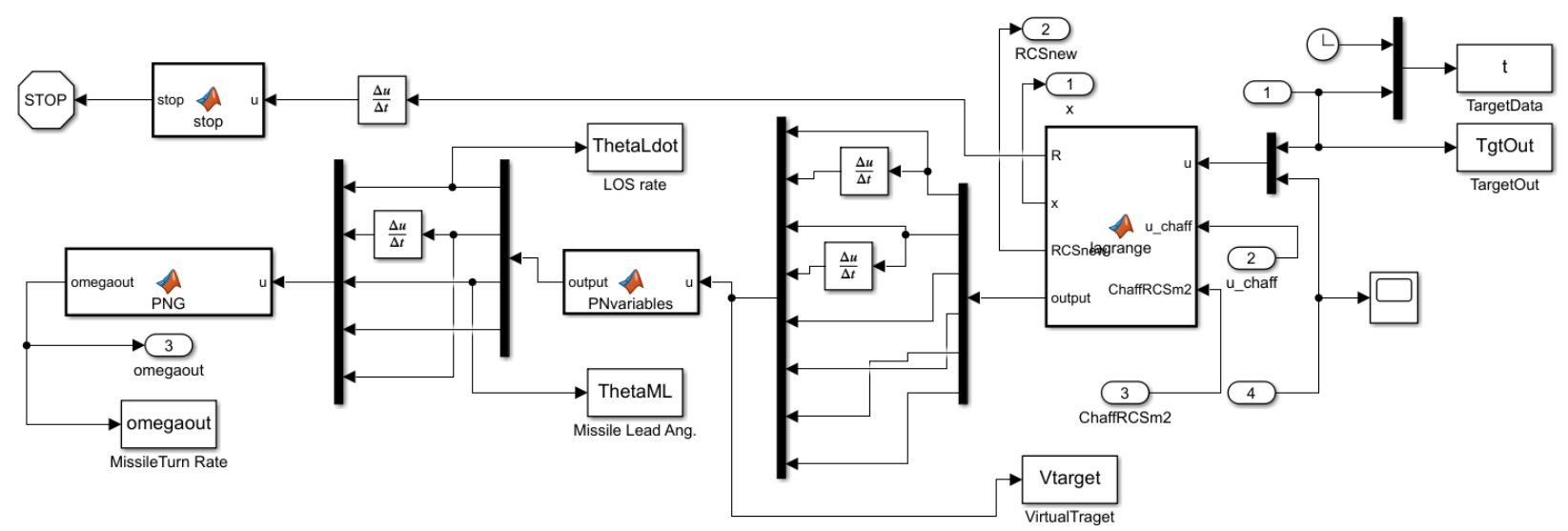

(d)

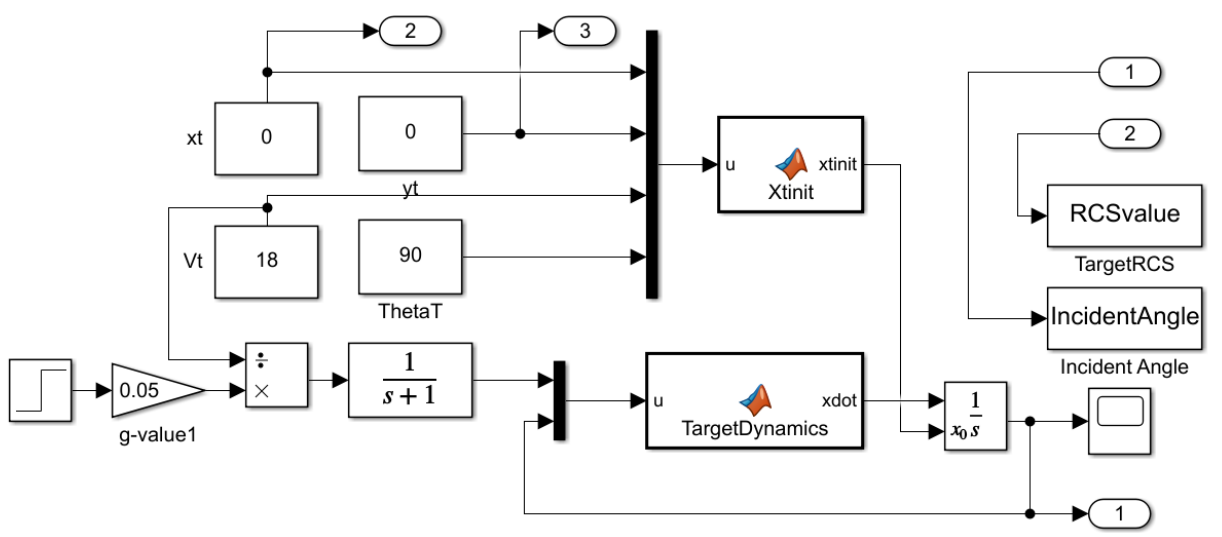

(e)

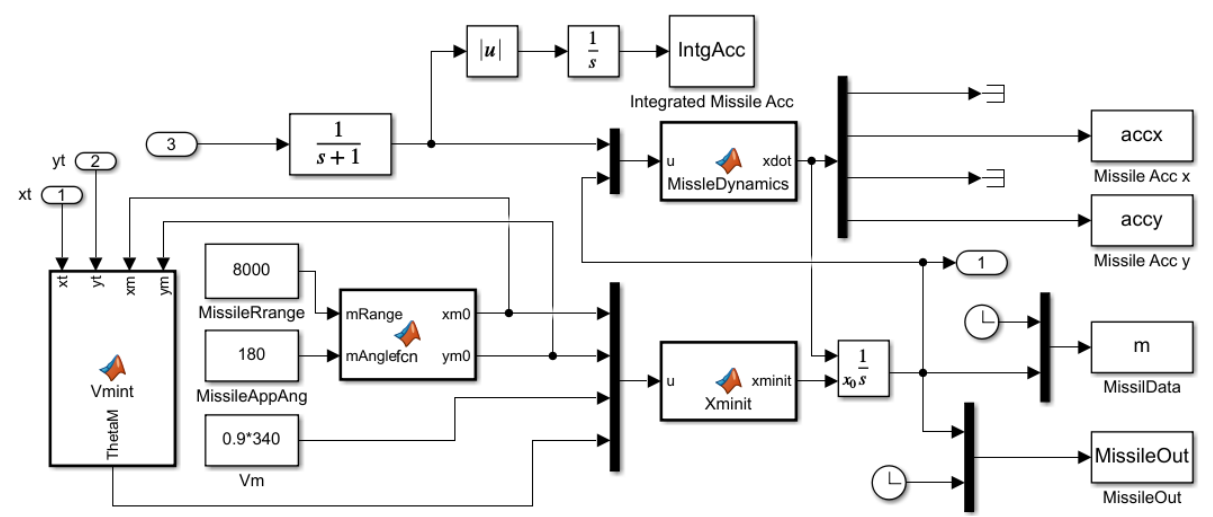

Fig. 10 ASMD/RCS block configuration: (a) ASMD/RCS, (b) decoy block, (c) guide block, (d) target ship block, and (e) missile block 


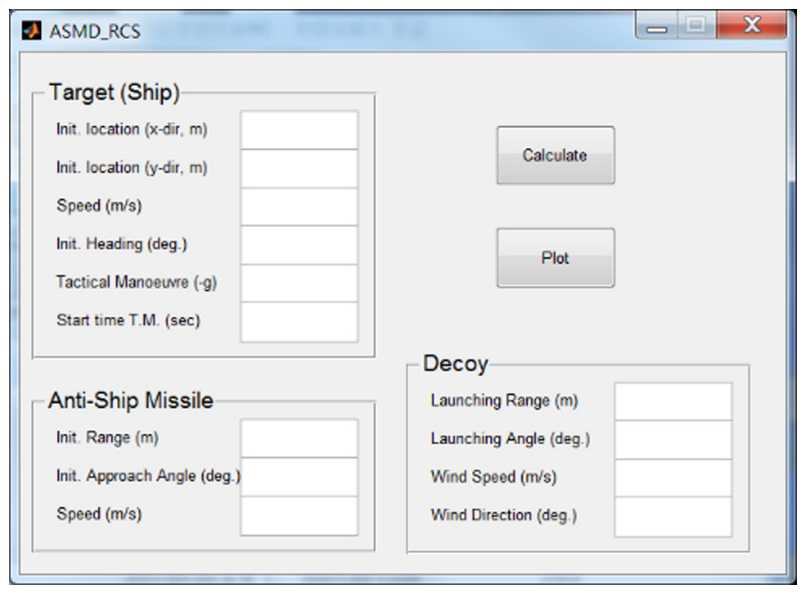

(a)

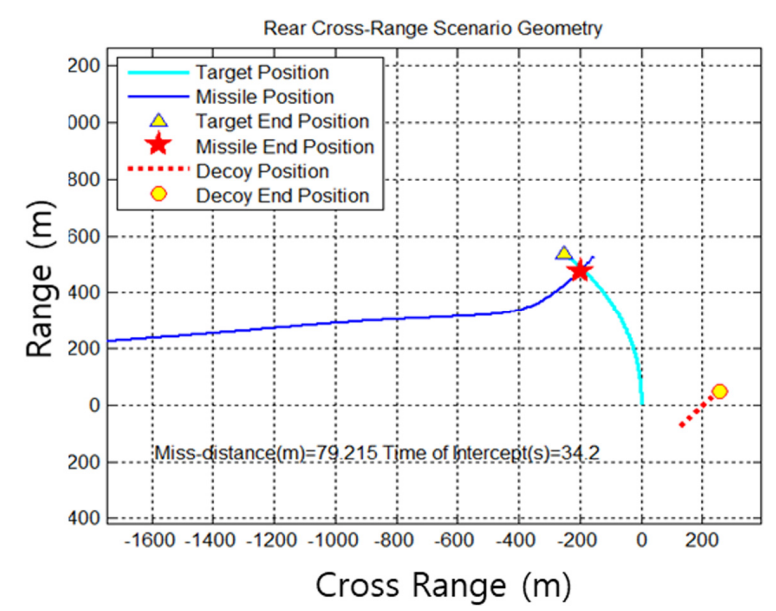

(b)

Fig. 11 ASMD/RCS (a) user interface and (b) engagement trajectory viewer

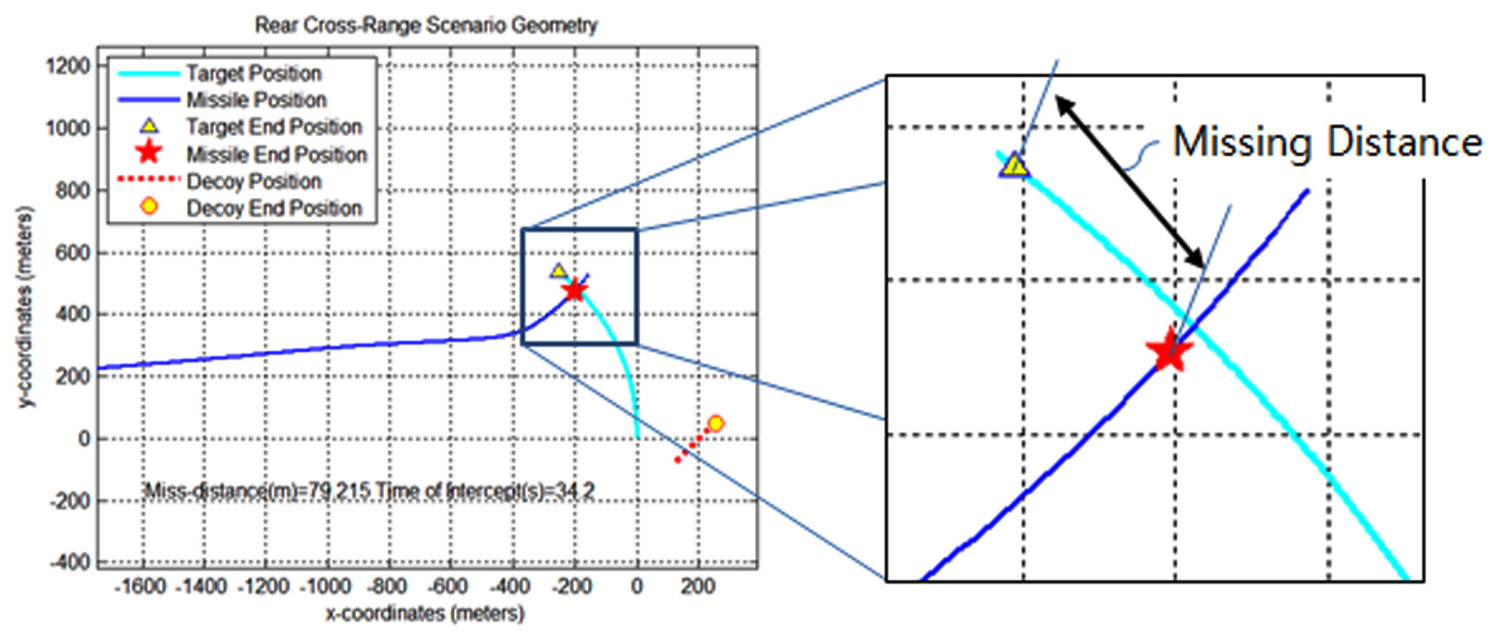

Fig 12 Definition of missing distance

position, RCS, speed, direction, and evasive maneuver of the target ship). Finally, the missile block simulates the ASM dynamics.

Fig. 11 shows the program's user interface and a plot of the simulation results. The interface is composed of 13 input boxes for simulation parameters and two buttons for program execution and a result plot. The plot displays trajectories of the ASM, the target ship, and the decoy, as well as text-based results, such as the missing distance and time to intercept. The term "missing distance" means the closest point of approach (CPA) synchronized from the center of the target ship to the ASM trajectory at the moment "time of intercept" (Fig. 12). This judges whether the target ship will be intercepted by the ASM or not by assessing whether the resultant missing distance in $m$ exceeds the pre-defined threshold. This study uses two times the length of the target ship as a threshold.

\section{Numerical Examples}

\subsection{Engagement Scenarios}

Decoy effect simulations were conducted using ASMD/RCS in some engagement scenarios. Following the input parameter symbols in
Fig. 13, the engaging speed $v_{m}$, lock-on range $R_{m}$, and approach angle $\theta_{m}$ of the ASM were set to Mach $0.9(\sim 306 \mathrm{~m} / \mathrm{s}), 8 \mathrm{~km}$, and 180 degrees, respectively. The centripetal acceleration was limited to 20 -g. The length of the target ship was $45 \mathrm{~m}$, and the corresponding missing distance threshold was two times the target ship length. The ship speed $v_{s}$ was 35 knots $(\sim 18 \mathrm{~m} / \mathrm{s})$, and the RCS pattern $\sigma_{s}$ is shown in Fig. 14. The decoy is launched toward any direction $\theta_{d}$ and deployed at a range $R_{d}$ of $150 \mathrm{~m}$.

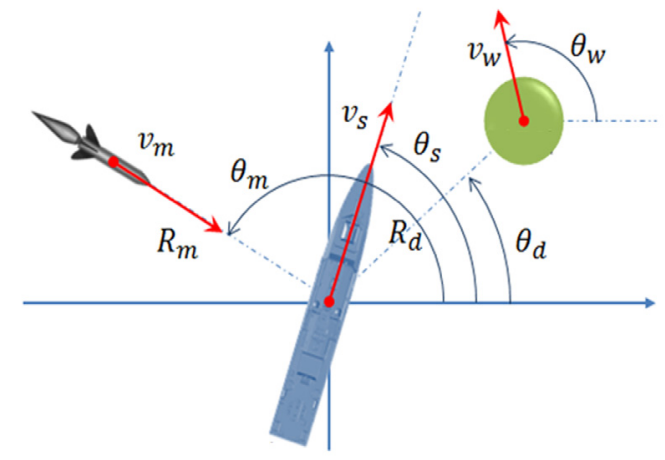

Fig. 13 Input parameter for engagement simulation 


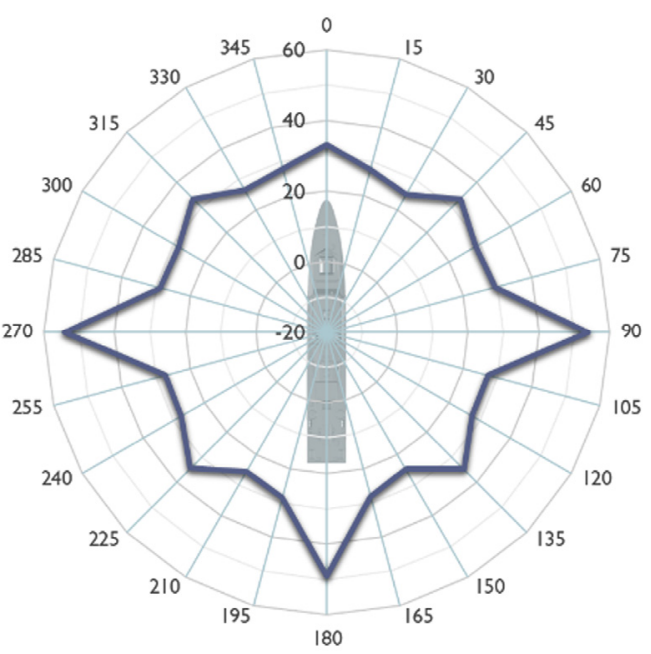

Fig. 14 RCS pattern of the target ship for engagement simulation

The RCS level $\sigma_{d}$ of the decoy varies $(27,30,33,37$, and $40 \mathrm{dBsm}$, which correspond to 500,1000, 2000, 5000, and $10000 \mathrm{~m}^{2}$, respectively). There is only one chance of launching a decoy, and an evasive maneuver is done during the engagement at the same time as the ASM lock-on. The simulation designated three engagement scenarios with varying g-value $G_{s}^{*}$ of the target ship to 0 -g, $0.05-\mathrm{g}$, and -0.05 -g when the wind speed $v_{w}$ and direction $\theta_{w}$ were 10 knots $(\sim 5.14$ $\mathrm{m} / \mathrm{s}$ ) and 45 degrees, respectively.

\subsection{Simulation Results}

Fig. 15 shows simulation results for the scenarios for when the decoy launching direction $\theta_{d}$ is 135 degrees and the RCS of the target ship is $40 \mathrm{dBsm}\left(10,000 \mathrm{~m}^{2}\right)$. The target ship survives when doing no evasive maneuver $(0-\mathrm{g})$, but it is intercepted when doing the port-side $(0.05-\mathrm{g})$ and starboard-side ( $-0.05-\mathrm{g})$ evasive maneuvers. In starboardside evasive maneuvers, the primary deception succeeds, but the ship is eventually intercepted. This means that ASMD/RCS can simulate complicated engagement scenarios more realistically.

Fig. 16 shows polar plots of the simulated missing distance versus the decoy launching direction $\theta_{d}$ for the scenarios. The curves present the simulated missing distance with respect to the RCS level $\sigma_{d}$ of the decoy $\left(27 \mathrm{dBsm}\left(500 \mathrm{~m}^{2}\right), 30 \mathrm{dBsm}\left(1,000 \mathrm{~m}^{2}\right), 33 \mathrm{dBsm}\left(2,000 \mathrm{~m}^{2}\right)\right.$, $37 \mathrm{dBsm}\left(5,000 \mathrm{~m}^{2}\right)$, and $\left.40 \mathrm{dBsm}\left(10,000 \mathrm{~m}^{2}\right)\right)$. The dotted red reference line shows the pre-defined threshold (two times the target ship length: $90 \mathrm{~m}$ ).

With no evasive maneuver $(0-\mathrm{g})$, the missing distance increased as the decoy RCS increased. When the decoy RCS levels were above 37 $\mathrm{dBsm}\left(5,000 \mathrm{~m}^{2}\right)$, the target ship was hit less overall. When the decoy of $33 \mathrm{dBsm}\left(2,000 \mathrm{~m}^{2}\right)$ was launched between 150 degrees and 300 degrees, the target ship was hit less. When the decoy RCS level was 40 $\mathrm{dBsm}\left(10,000 \mathrm{~m}^{2}\right)$, wind reduced the susceptibility of the 300-degree and 330-degree decoy deployments.

For the port-side evasive maneuver $(0.05-\mathrm{g})$, the decoy effect was minimal in general. An evasive maneuver works unfavorably in terms of susceptibility to ASMs. The decoy was moved by the wind along

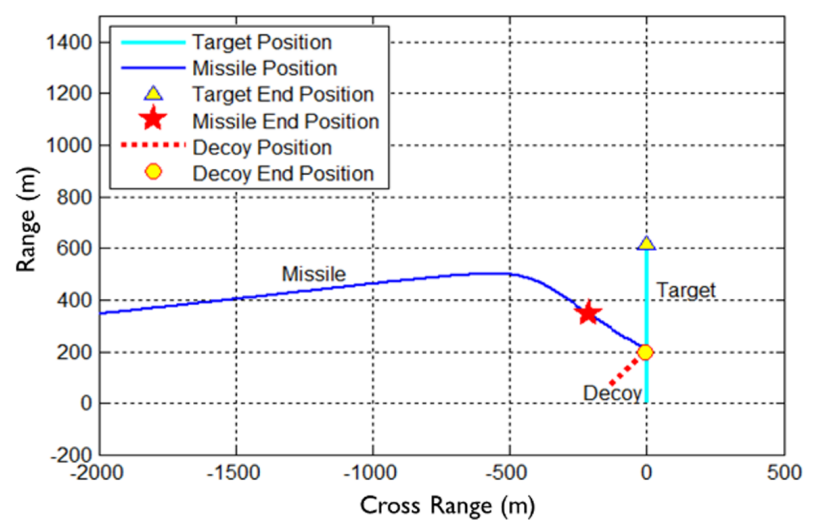

(a)

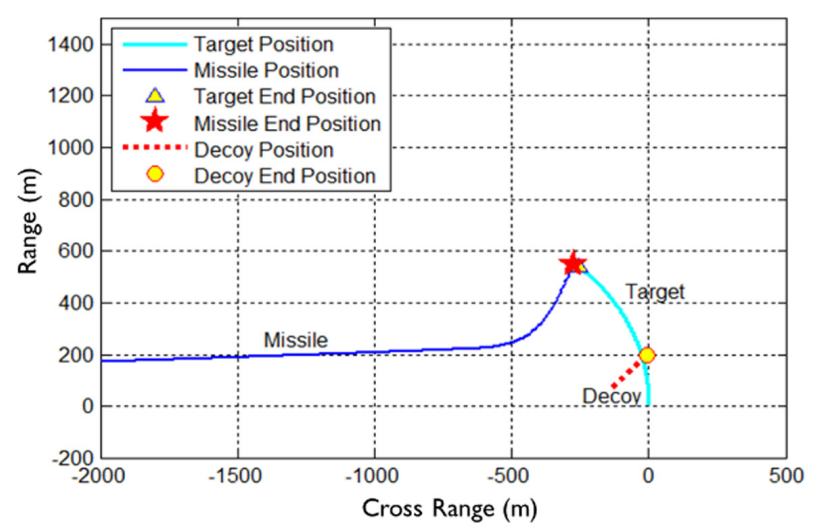

(b)

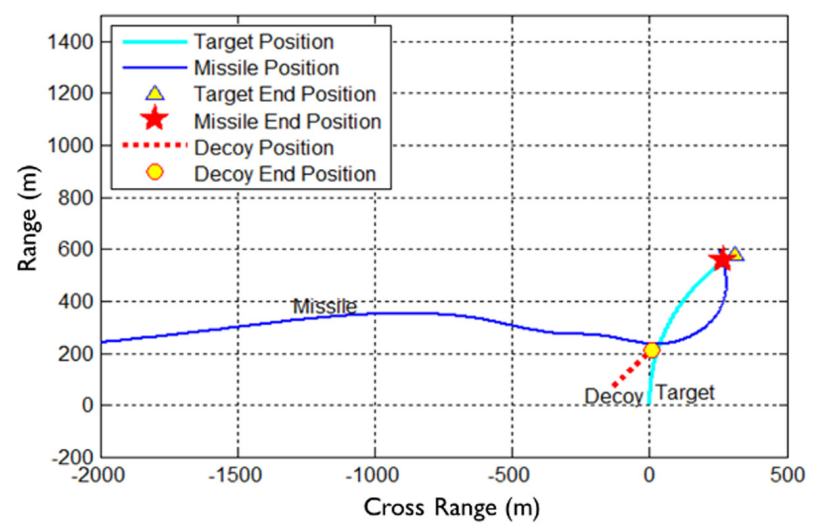

(c)

Fig. 15 Engagement simulation results when the decoy launching direction $\theta_{d}$ is 135 degrees: (a) no evasive maneuver $(0-\mathrm{g})$, (b) port-side evasive maneuver $(0.05-\mathrm{g})$, and (c) starboardside $(-0.05-\mathrm{g})$ evasive maneuver

the 45-degree direction, which slightly increased the susceptibility of the decoy deployments at 0 degrees, 300 degrees, and 330 degrees. For the starboard-side evasive maneuver $(-0.05-\mathrm{g})$, the missing distance increased as the decoy RCS increased. Simultaneously, the susceptibility was reduced when the decoy was deployed toward 120 to 240 degrees. It was confirmed that the ASMD/RCS program was successfully implemented and could be applied to decoy effect simulations of various engagement scenarios. 


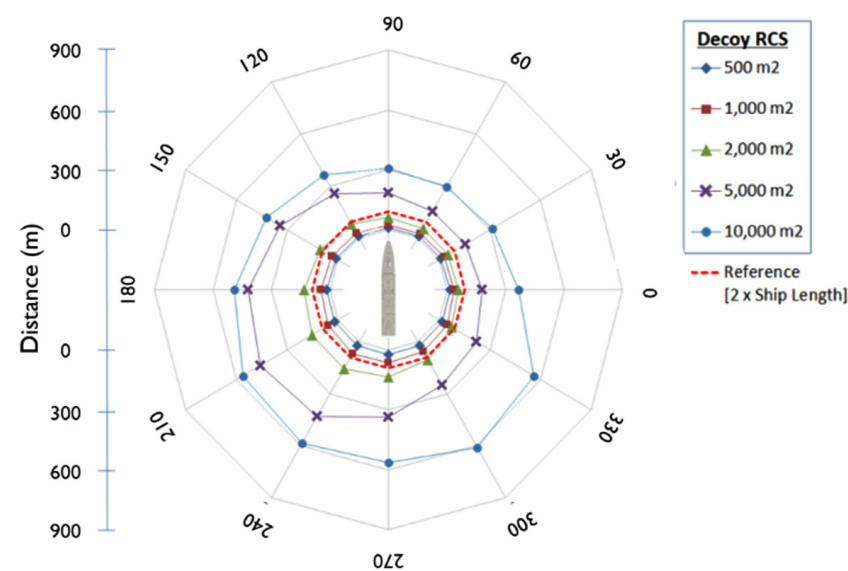

(a)

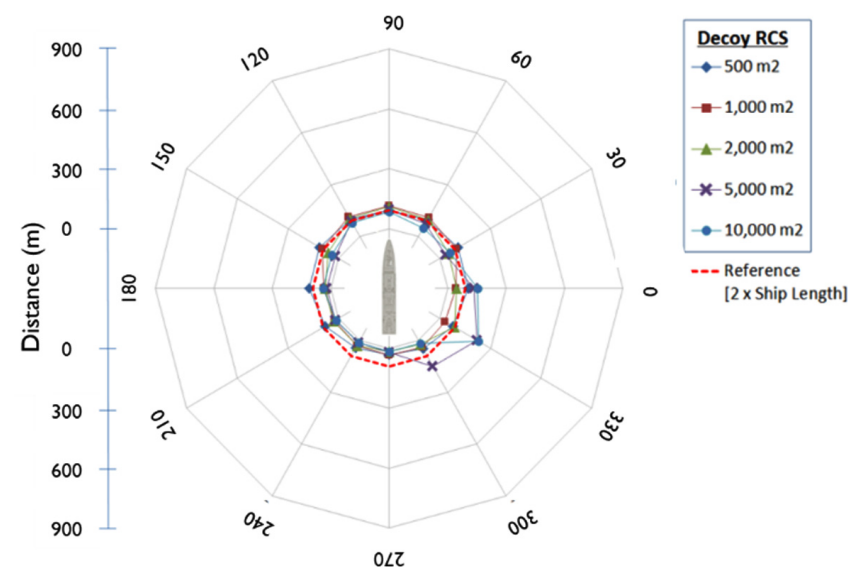

(b)

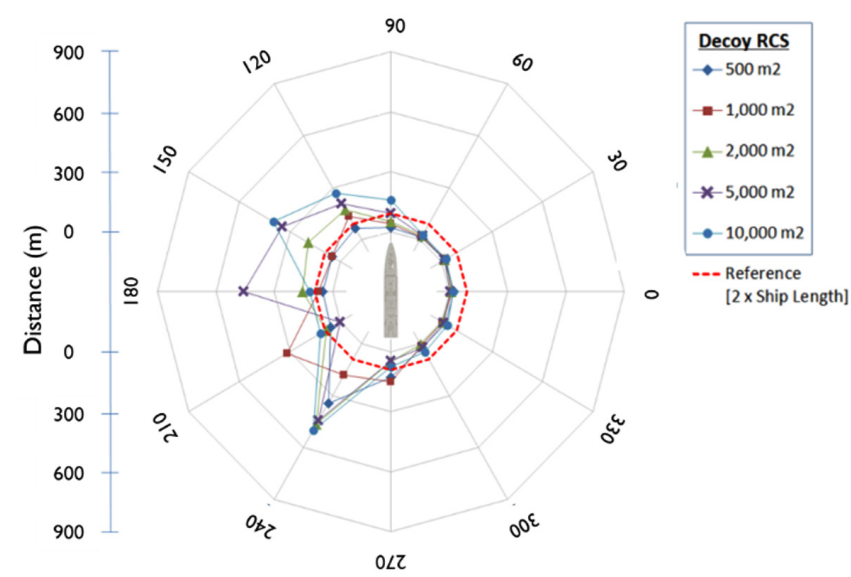

(c)

Fig. 16 Simulated missing distance versus the decoy launching direction $\theta_{d}$ in engagement scenarios: (a) no evasive maneuver $(0-\mathrm{g})$, (b) port-side evasive maneuver $(0.05-\mathrm{g})$, and (c) starboard-side evasive maneuver $(-0.05-\mathrm{g})$.

\section{Conclusions}

An engagement scenario-based simulation program named ASMD/RCS was developed based on a state-space model to assess the decoy effect on a sea-skimming ASM using an active radar homing for targeting and tracking. The programs can consider the RCS and tactical maneuvers of the target ship, the onboard decoy system performance, the ASM guidance algorithm, and the engagement environment's wind speed and direction. The program provides a user interface for input parameters, executing the program, and plotting results. Numerical simulations of virtual engagement scenarios were conducted for various RCSs and launching directions of the decoy. The results showed that the input parameter variations in engagement environments influence the decoy effect. It is expected that the program could be practically used for assessing a decoy system's performance for naval ships.

\section{Acknowledgments}

This research was supported by Tongmyong University Research Grants 2019 (2019A020-1).

\section{References}

An, B., \& Seo, S. (2015). A Study on the Setting Procedure of Standard Value and Design Target Value for the RCS Reduced Design for Naval Ships. Journal of Korean Institute of Electromagnetic Engineering and Science, 26(6), 581-588. https://doi.org/ 10.5515/KJKIEES.2015.26.6.581

Chapman, S.J., \& Benke, K. (2000). Assessment of Ship Air Defence Performance by Modelling and Simulation. Proceedings of the Simulation Technology and Training Conference, Perth WA, Australia, 235-241.

Dan, J., Hao, C., \& Lei, Z. (2016). A Simulation Study of Chaff Echo Signal Based on LFM. International Journal of Signal Processing, Image Processing and Pattern Recognition, 9(4). 131 -140. https://doi.org/10.14257/ijsip.2016.9.4.12

Kim, K., \& Lee, J. (2014). Integrated Survivability Assessment Given Multiple Penetration Hits. Journal of Ocean Engineering and Technology, 28(1), 69-76. https://doi.org/10.5574/KSOE.2014. 28.1.069

Kim, K. (2020). Decoy Effectiveness Simulation Against Anti-Ship Missile Considering RCS and Tactical Diameter of Warship. Proceeding of Korean Society of Ocean Engineers Fall Conference, On-line, 198-200.

Kumar, R. (1990). Defense of Surface Ships Against Anti-Ship Missiles (Master's thesis). Naval Postgraduate School, Monterey CA, USA.

Lukenbill, F.C. (1990). A Target Missile Engagement Scenario Using Classical Proportional Navigation (Master's thesis). Naval Postgraduate School, Monterey CA, USA.

Manji, N., Kocakanat, M., \& Kitsikis, A. (2002). Quantifying the Effects of Chaff Screening on Hardkill and Softkill Coordination (Report No. RTO-MP-097(I)). Ottawa Canada: Defense R\&D Canada. 
Poulos, A.M. (1994). An Anti-air Warfare Study for a Small Size Navy (Master's thesis). Naval Postgraduate School, Monterey CA, USA.

TTI. (2002). User's Guide: ASCMA Anti-Ship Cruise Missile ECM Effectiveness Simulator. 3.2, Ottawa, Ontario, Canada: Tactical Technologies Inc.

Seo, D.W., Nam, H., Kwon O., \& Myung, N.H. (2012). Dynamic RCS Estimation of Chaff Clouds. IEEE Transactions on Aerospace and Electronic Systems, 48(3), 2114-2127. https://doi.org/10. 1109/TAES.2012.6237582
Swee, J. CS. (2000). Missile Terminal Guidance and Control Against Evasive Target (Master's thesis). Naval Postgraduate School, Monterey CA, USA.

\section{Author ORCID}

Author name

Kim, Kookhyun
ORCID

0000-0002-4214-4673 